\title{
Palmprint Recognition Based on Directional Features and Graph Matching
}

\author{
Yufei Han, Tieniu Tan, and Zhenan Sun \\ Center for Biometrics and Security Research \\ National Labrotory of Pattern Recognition,Institue of Automation \\ Chinese Acdamey of Sciences \\ P.O. Box 2728, Beijing, P.R. China, 100080 \\ \{yfhan, tnt, znsun\} @nlpr.ia.ac.cn
}

\begin{abstract}
Palmprint recognition, as a reliable personal identity check method, has been receiving increasing attention during recent years. According to previous work, local texture analysis supplies the most promising framework for palmprint image representation. In this paper, we propose a novel palmprint recognition method by combining statistical texture descriptions of local image regions and their spatial relations. In our method, for each image block, a spatial enhanced histogram of gradient directions is used to represent discriminative texture features. Furthermore, we measure similarity between two palmprint images using a simple graph matching scheme, making use of structural information. Experimental results on two large palmprint databases demonstrate the effectiveness of the proposed approach.
\end{abstract}

\section{Introduction}

Biometrics identifies different people by their physiological and behavioral difference, such as face, iris, retinal, gait, etc [1]. As an alternative personal identity authentication method, it has attracted increasing attention during recent years. In the field of biometrics, palmprint is a novel but promising member. Most discriminating patterns of palmprint could be captured by low resolution capture devices, such as a low-cost CCD camera [3]. Large region of palm supplies stable line patterns which are difficult to be faked.

A key issue in palmprint analysis is finding a proper descriptor to represent its line patterns [5]. In previous work, local texture based approach is proved to be the most efficient $[1,2,3,4,5,6]$. Since line patterns of palmprint are always spread over different image areas, both description of local patterns and their spatial relation are important for describing palmprint in an accurate way. Therefore, component based image representation supplies a reasonable framework, following which we could design efficient palmprint recognition methods by adopting local image features.

In this paper, we introduce a novel approach for palmprint recognition which considers both texture information of local image regions and the spatial relationships between these regions. In our method, a straightforward extraction of image gradients vector is adopted across the whole palmprint image plane. Derived vector field is then divided into blocks. Statistical texture features of each block are encoded by a local 
direction histogram (LDH) proposed in this paper, which describes distribution properties of the directional vectors. All localized blocks form a global graph. The similarity of two palmprint images is measured by a simple graph matching method, previously utilized in [9].

The remainder of this paper is organized as follows. Details about LDH scheme and the graph matching method are described in section 2 and 3 respectively. In section 4, experimental results are reported. Section 5 concludes the paper.

\section{Local Direction Histogram Based Palmprint Description}

Image gradient has been used as an efficient indicator of local image texture [8]. As reported in [7], it has a close relation with reflectance and surface normal of objects imaged, which decide inherent structures of corresponding image patches. Benefit from it, histogram of gradient directions has been widely used as a powerful image descriptor [10][11]. It captures characteristic patterns of local image region. In our work, we utilize histograms of oriented gradients to represent texture information of palmprint images.

We calculate gradient vectors by the first order Gaussian derivative operator $\nabla G$, in order to avoid noise sensitivity caused by directly differentiation on original intensities. It should be noted that size of $\nabla G$ operator is chosen according to needs of applications. A proper choice makes good trade-off between noise tolerance and locality of description. For a local image region $W$, the gradient magnitude $M$ and direction angle $\theta$ are expressed respectively as follows:

$$
\begin{aligned}
& M=\sqrt{\left(\nabla G_{x} * W\right)^{2}+\left(\nabla G_{y} * W\right)^{2}} \\
& \theta=\tan ^{-1}\left(\frac{\nabla G_{y} * W}{\nabla G_{x} * W}\right)
\end{aligned}
$$

The whole procedure of palmprint feature extraction is illustrated in Fig.1. Before feature extraction and matching, input palmprint images are firstly normalized to regulate translation, rotation and scale variation among different palms, following the method employed in [3]. In our work, assuming the size of normalized palmprint image and $\nabla G$ operator is $N$ by $N$ and $m$ by $m$ respectively. After calculation of gradient vector on each sample region, the size of derived vector valued image is $(N$ $m+1)$ by $(N-m+1)$. To focus on local texture representation, we divide the whole vector field into $r^{*} r$ square blocks, each of them has the side length of $(N-m+1) / r$.

For each block, we again divide it into 4 sub-regions with the same size. A 6-bin direction histogram is constructed covering the 360 degree range of gradient directions in each sub-region. Same as in [10], samples are added to the histogram, weighted by corresponding gradient magnitudes. This histogram contains information about distribution of the direction angles, which represents details of image structure in a statistical way. Besides, spatial information is also important for more efficient representation. So we combine histograms of these four neighboring regions to yield a 24-bin spatially enhanced histogram and normalize it to unit length, namely, local direction histogram, as shown in Fig. 2. Through this way, local statistical descriptions and their spatial arrangement are concatenated into a more discriminative 
texture feature of the block region. A more involve method to construct histograms is based on adding gradient vectors into bins with equal weights, rather than weighted by their magnitudes. The gradient magnitude is sensitive to changes in illumination settings, such as direction changes of light sources. In contrast, the direction angle is more stable under those variations. Through this scheme, we can improve robustness of texture representation. Both two types of histogram based features are evaluated in the experiment.

Finally, we take each block as a node of a graph map, associated with a local direction histogram, illustrated also in Fig.1. As a result, a palmprint image is represented by such a map in two different respects. Bins of local direction histograms represent statistical characteristics of texture patterns on the region level. Topology relations among nodes describe spatial layouts of blocks and thus produce a more global description of the whole image.
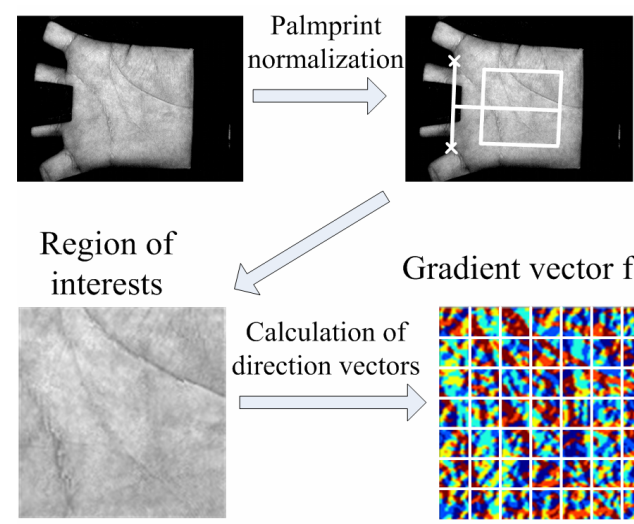

Gradient vector field

Calculation of direction vectors
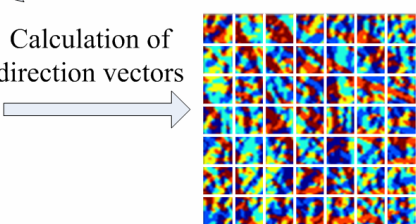

Graph based representation

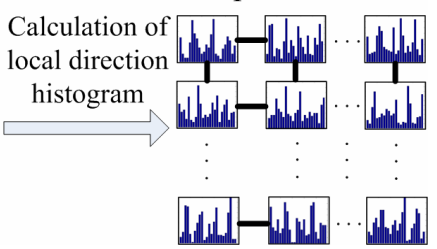

Fig. 1. Diagram of palmprint feature extraction

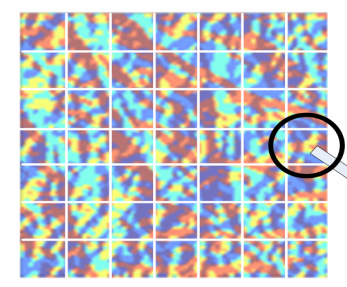

Gradient vector field

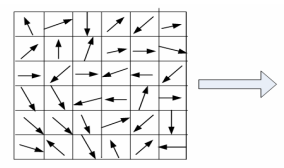

Sub-region A

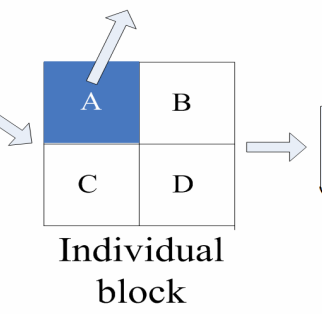

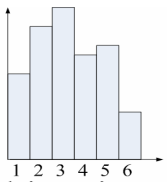

6-bin Direction

Histogram

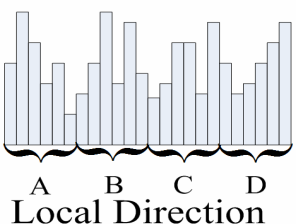

Histogram

Fig. 2. Generation of local direction histogram 


\section{Graph Matching Approach}

Each node of the graph representation has two attributes, local direction histogram feature associated with it, and its spatial relation with other nodes. It is a natural idea to measure similarity of two palmprint images by comparing nodes of corresponding graphs. In our work, we adopt a simple graph matching algorithms proposed by Sun et al [9]. Each node of one palmprint image is supposed to be the most similar with the corresponding one of another palmprint image, if these two are captured from the same palm. Similarly in [9], before any discussion, we define conditions that matching nodes should satisfy.

Assuming the graph representations of two palmprint images are $\left\{A_{i}\right\}$ and $\left\{B_{j}\right\}$ $(i, j=1,2 \ldots S)$ respectively, two matching nodes should have the same spatial position in each graph. Furthermore, texture patterns of matching node pair $A_{i}$ and $B_{i}$ should be most similar under a certain metric among all pairs composed by $A_{i}$ and individual $B_{j}(i, j=1,2 \ldots S)$. Therefore, we count the number of matching pairs to evaluate resemblance between two images. The higher it is, the more likely the two images come from the same palm. Through this kind of procedure, we make full use of both texture and structure properties of each node to achieve accurate classification.

In our paper, we utilize the Chi-square distance between local direction histograms $\left\{L D H A_{i}\right\}$ and $\left\{L D H B_{j}\right\}(i, j=1,2 \ldots S)$ to measure texture similarity of two nodes:

$$
\chi^{2}\left(L D H A_{i}, L D H B_{j}\right)=\sum_{k=1}^{24} \frac{\left(L D H A_{i}^{k}-L D H B_{j}^{k}\right)^{2}}{L D H A_{i}^{k}+L D H B_{j}^{k}}
$$

Based on this metric, Chi-square distance between histogram features of two matching nodes should be lower than a prefixed threshold [9]. Following pseudo codes illustrate how to compute the number of matching pairs $N$ step by step:

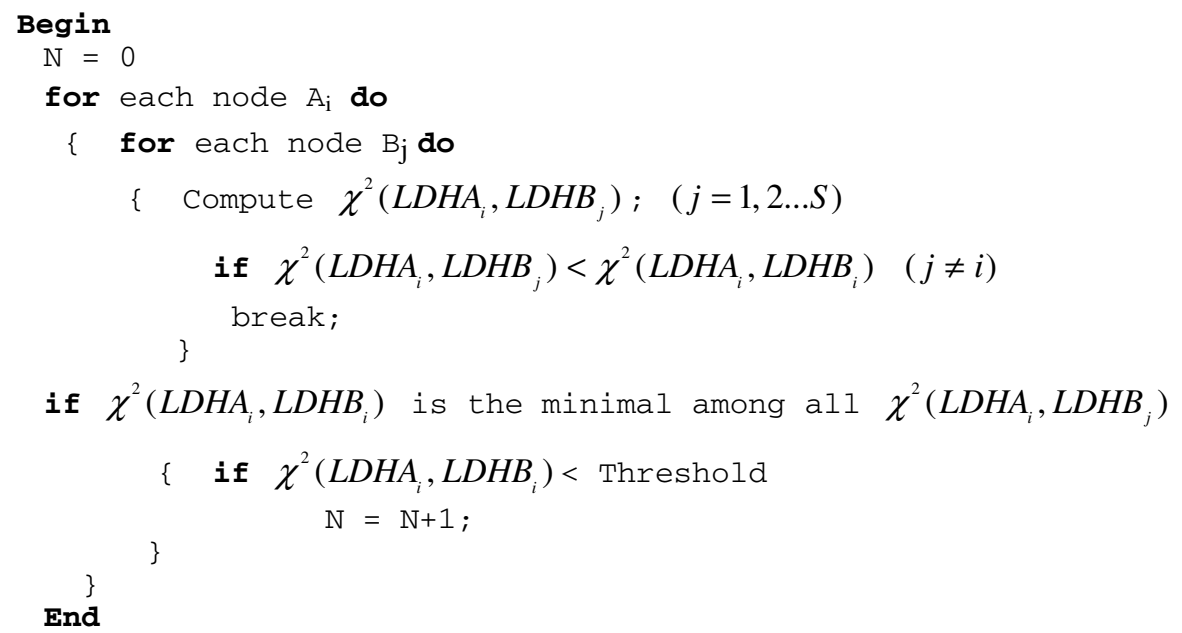


To obtain normalized matching score ranging between 0 and 1, we can divide $N$ by $S$, the total number of nodes in each graph map. In the following experiment, we directly employ $N$ as the matching score for convenient.

\section{Verification Experiments}

In this section, we test the performance of the proposed approach on the PolyU Palmprint Database [12] and the CASIA Palmprint Database [13]. In the first dataset, images which belong to the same palm contain deformation of skin surface, such as contraction and stretching (see Fig. 7). Images in the second one are mostly captured with less such variations between intra-class samples.

\subsection{Verification Experiment on the PolyU Palmprint Database}

The PolyU database [12] contains 7,752 palmprint images from 386 palms. Each palm has two sessions of images. Average time interval between two sessions is two months. Light conditions and focus of the imaging device are changed between two occasions of image capturing [3], which is challengeable to robustness of recognition algorithms. After preprocessing, regions of interests, with the size of $128 \times 128$ are obtained. The $\nabla G$ operator has the size of 9 by 9 . After gradient calculation, the whole vector valued image is divided into $6 * 6=36$ blocks. All images are used to estimate intra-class distribution. We select five images randomly from each session to form inter-class samples. If a session contains less than five images, we make up totally ten samples from the class which the session belong to, including all images contained in the session. Therefore, 74,068 intra-class matching and 7,430,500 inter-class matching are involved in the experiment. In this experiment, we evaluate two LDH based methods. The first constructs histograms with samples weighted by their corresponding gradient magnitudes, while the second treats each sample with the same weight. The other three state-of-the-art algorithms, namely, fusion code [4], competitive code [5], ordinal code [6], are implemented for further comparing. Experimental results are denoted in Table 1 and Fig. 3.

Table 1. Comparisons of performances on the PolyU database

\begin{tabular}{|l|l|l|}
\hline Algorithm & EER [14] & $d^{\prime}[14]$ \\
\hline Fusion code [4] & $0.21 \%$ & 5.40 \\
\hline Competitive code [5] & $0.04 \%$ & 5.84 \\
\hline Ordinal code [6] & $0.05 \%$ & 6.90 \\
\hline Weighted LDH & $0.10 \%$ & 7.13 \\
\hline Non-weighted LDH & $0.08 \%$ & 6.12 \\
\hline
\end{tabular}




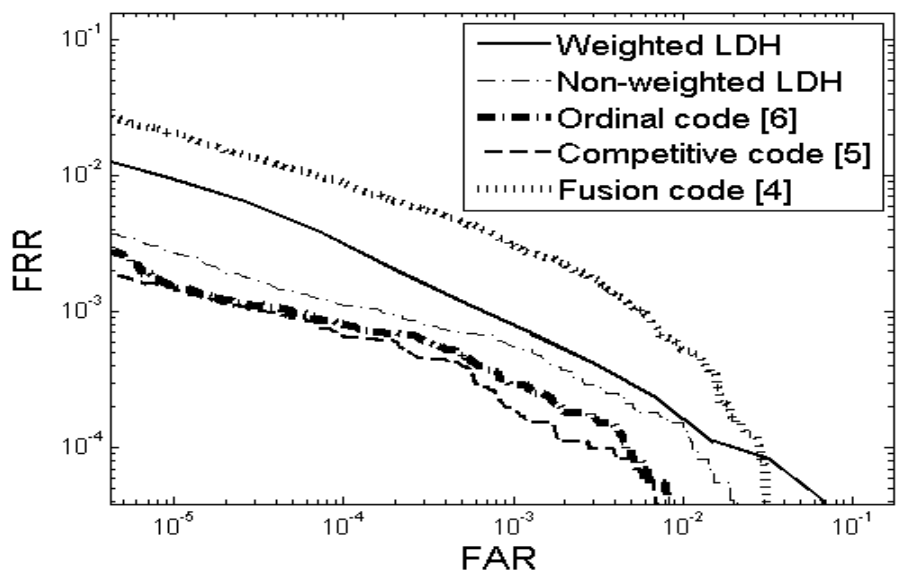

Fig. 3. ROC curves on the PolyU database

We named the first method "Weighted LDH" and the second " Non-weighted LDH". As shown in Fig. 3, in terms of ROC curves, both of LDH based methods achieve comparable performances, compared with ordinal code [6] and competitive code [5]. Notably, they are more accurate than Fusion code [4]. Of the two proposed approaches, non-weighted LDH performs obviously better, which is more robust to intra-class appearance variations.

\subsection{Verification Experiments on the CASIA Palmprint Database}

The CASIA Palmprint database [13] contains 4,512 24-bit color palmprint images, coming from 564 palms. Each palm has 8 images. During image capturing, palms of subjects are required to be laid on a uniform-colored background (see in Fig. 4(a)). Then palmprint images are captured by a common CMOS camera above palms. There are no pegs to restrict postures and positions of palms. Original size of each image is $640 \times 480$. After preprocessing, we crop a square region with size of $176 \times 176$, as region of interests (ROI), show in Fig. 4(b). We again adopt $9 \times 9 \nabla G$ operator and divide the whole vector valued image into $6^{*} 6=36$ blocks. All possible intra-class samples are used to simulate genuine distribution. One image is selected randomly from each class to estimate imposter distribution. Thus, totally 15,792 intra-class comparisons and 158,766 inter-class comparisons are implemented.

Tab 2 and Fig. 5 illustrate performances of two proposed LDH methods and the other three state-of-the-art algorithms. As we see, non-weighted LDH method achieves the highest accuracy, followed by weighted LDH, ordinal code [6], competitive code [5] and fusion code [4]. Compared with results in section 4.1, both two LDH based approaches perform better. That 's because that deformation of skin surface (see Fig.7) existing in intra-class samples of the PolyU database [12] changes reflectance and geometrical structures of skin surface, which then declines similarities of local distributions of image gradient directions. 

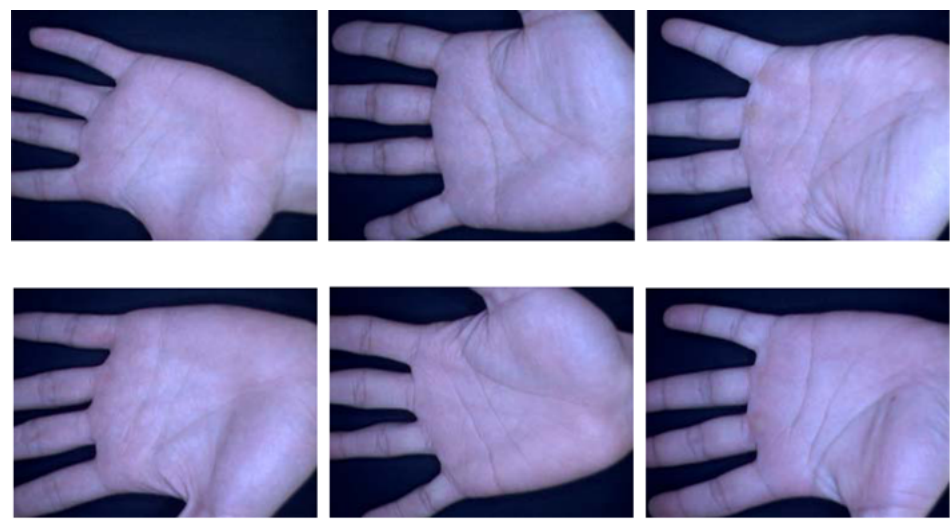

(a)
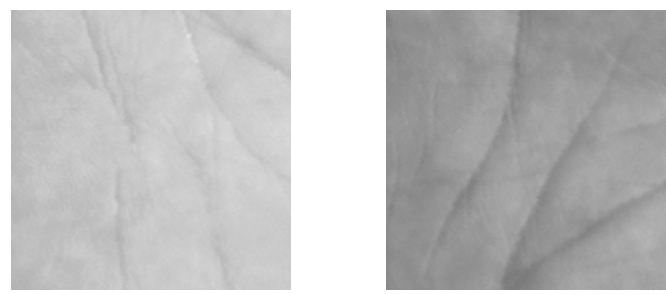

(b)

Fig. 4. (a) Palm images in the CASIA database (b) Cropped ROI

Table 2. Comparisons of performances on the CASIA database

\begin{tabular}{|l|l|l|}
\hline Algorithm & EER [14] & $d^{\prime}[14]$ \\
\hline Fusion code [4] & $0.57 \%$ & 3.73 \\
\hline Competitive code [5] & $0.19 \%$ & 3.82 \\
\hline Ordinal code [6] & $0.08 \%$ & 5.65 \\
\hline Weighted LDH & $0.05 \%$ & 9.30 \\
\hline Non-weighted LDH & $0.04 \%$ & 10.51 \\
\hline
\end{tabular}

Furthermore, Fig. 6 describes the intra-class and inter-class matching score distributions using non-weighted LDH on the PolyU [12] and the CASIA [13] databases. As shown in the figure, for most intra-class samples, the number of matching block pairs is higher than ten, about $1 / 3$ of the total blocks. Therefore, our approach only needs a fraction of the whole image area to deliver a valid classification. It can be used to handle palmprint images containing regions of occlusion or impaired palm skin. Compared with it, state-of-the-art algorithms [4][5][6] achieve successful recognition requiring most of the image region should be matched. 


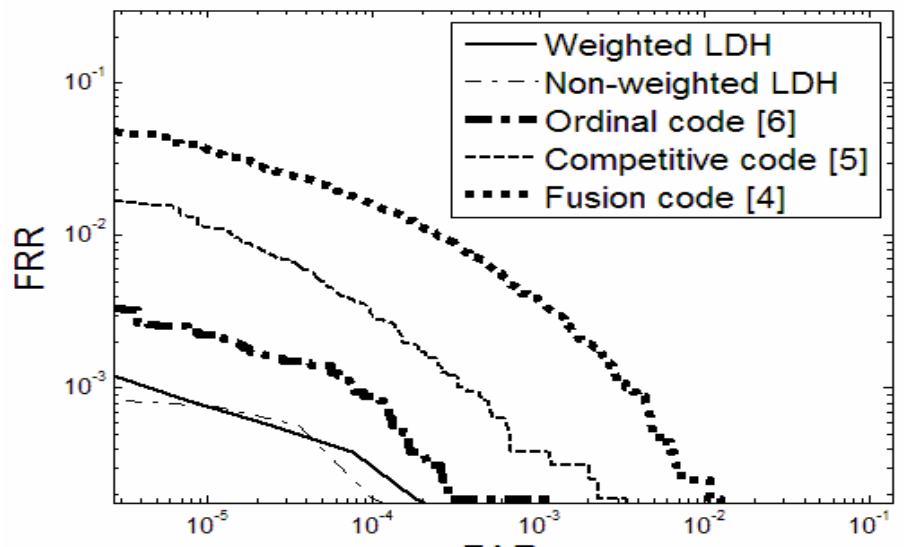

FAR

Fig. 5. ROC curves on the CASIA database

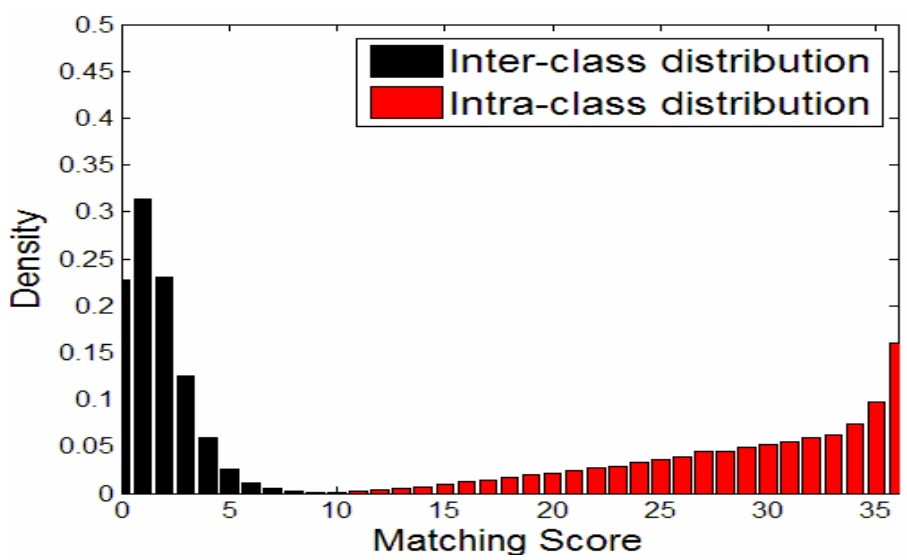

(a)

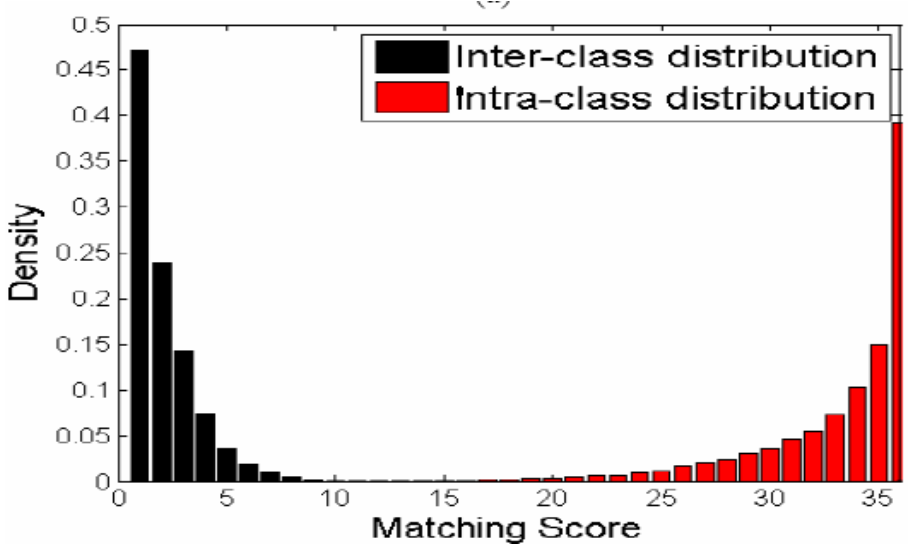

(b)

Fig. 6. Distribution of matching scores on the PolyU (a) and the CASIA (b) databases 

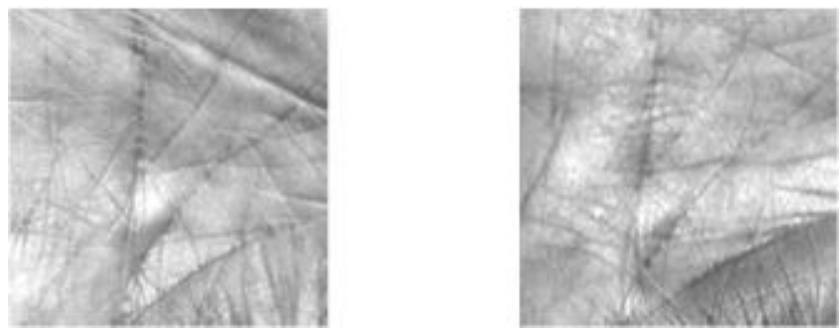

Fig. 7. Deformation of skin surface in the PolyU database

\section{Conclusions}

In this paper, we have proposed a novel palmprint recognition method by utilizing LDH based local texture descriptor and graph matching. It involves three main parts, namely, gradient vector calculation, local direction histogram generation and graph matching. By organizing local texture features to form a graph based representation, we can differentiate two palmprint images from both fine details and global structural information through a simple graph matching procedure. Our extensive experimental results have demonstrated validity of the approach. In our method, it is an important issue to choose a proper number of histogram bins and size of local image blocks. However, this problem is still not well addressed in our paper and needs further work in the future. In a further step, we will investigate how to find more efficient structural features to improve descriptive power of palmprint representations.

Acknowledgments. Experiments in the paper use the PolyU Palmprint Database $2^{\text {nd }}$ collected by the Biometric Research Center at the Hong Kong Polytechnic University. This work is funded by research grants from the National Basic Research Program (Grant No. 2004CB318110), the Natural Science Foundation of China (Grant No. 60335010, 60121302, 60275003, 60332010, 69825105, 60605008) and the Chinese Academy of Sciences.

\section{References}

1. Kong, W.K., Zhang, D., Li, W.X.: Palmprint feature extraction using 2-D Gabor filters. Pattern recognition 36, 2339-2347 (2003)

2. You, J., Li, W.X., Zhang, D.: Hierarchical palmprint identification via multiple feature extraction. Pattern recognition 35(4), 847-859 (2002)

3. Zhang, D., Kong, W.K., You, J., Wong, M.: Online Palmprint Identification. IEEE Trans on PAMI 25(9), 1041-1050 (2003)

4. Kong, W.K., Zhang, D.: Feature-Level Fusion for Effective Palmprint Auentication. In: Zhang, D., Jain, A.K. (eds.) ICBA 2004. LNCS, vol. 3072, pp. 520-523. Springer, Heidelberg (2004)

5. Kong, W.K., Zhang, D.: Competitive Coding Scheme for Palmprint Verification. In: Proc.of the 17th ICPR, vol. 14, pp. 520-523 (2004) 
6. Sun, Z.N., Tan, T.N., Wang, Y.H., Li, S.Z.: Ordinal Palmprint Representation for Personal Identification. In: Proc. of CVPR 2005, vol. 1, pp. 279-284 (2005)

7. Chen, H.F., Belhumeur, P.N., Jacobs, D.W.: In Search of Illumination Invariants. In: Proc. of CVPR 2000, vol. I, pp. 254-261 (2000)

8. Sun, Z.N., Tan, T.N., Wang, Y.H.: Robust Direction Estimation of Graident Vector Field for Iris Recognition. In: Proc. of ICPR 2004, vol. 2, pp. 783-786 (2004)

9. Sun, Z.N., Tan, T.N., Qiu, X.C.: Graph Matching Iris Image Blocks with Local Binary Pattern. In: Zhang, D., Jain, A.K. (eds.) Advances in Biometrics. LNCS, vol. 3832, pp. 366-372. Springer, Heidelberg (2005)

10. Lowe, D.G.: Distinctive Image Features from Scale-Invariant Keypoints. International Journal of Computer Vision(IJCV) 60, 90-110 (2004)

11. Dalal, N., Triggs, B.: Histograms of Oriented Gradients for Human Detection. In: Proc.of CVPR 2005, vol. 1, pp. 863-886 (2005)

12. PolyU Palmprint Database, http://www.comp.polyu.edu.hk/ biometrics/

13. CASIA Palmprint Database, http://www.cbsr.ia.ac.cn

14. Daugman, J., Williams, G.: A Proposed Standard for Biometric Decidability. In: Proc. CardTech/SecureTech Conference, Atlanta, GA, pp. 223-234 (1996) 